J. Dairy Sci. 95:2157-2164

http://dx.doi.org/10.3168/jds.2011-4494

(C) American Dairy Science Association ${ }^{\circledR}, 2012$.

\title{
Short communication: Principal components and factor analytic models for test-day milk yield in Brazilian Holstein cattle
}

\author{
A. B. Bignardi, ${ }^{\star 1}$ L. El Faro,† G. J. M. Rosa,‡ V. L. Cardoso,† P. F. Machado,§ and L. G. Albuquerque*\# \\ *Department of Animal Science, São Paulo State University (FCAV/UNESP), 14884-900, Jaboticabal-SP, Brazil \\ †Agência Paulista de Tecnologia dos Agronegócios - APTA, Pólo Regional Centro Leste, 14075-310, Ribeirão Preto-SP, Brazil \\ ‡Department of Dairy Science, University of Wisconsin, Madison 53706 \\ $\S$ Department of Animal Science, University of São Paulo, 13418-900, Piracicaba, SP, Brazil \\ \#Conselho Nacional de Desenvolvimento Científico e Tecnologico (CNPq) and Instituto Nacional de Ciência e Tecnologia-Ciência Animal \\ (INCT-CA) 36570-000, Viçosa-MG, Brazil
}

\section{ABSTRACT}

A total of 46,089 individual monthly test-day (TD) milk yields (10 test-days), from 7,331 complete first lactations of Holstein cattle were analyzed. A standard multivariate analysis (MV), reduced rank analyses fitting the first 2, 3, and 4 genetic principal components (PC2, PC3, PC4), and analyses that fitted a factor analytic structure considering 2, 3, and 4 factors (FAS2, FAS3, FAS4), were carried out. The models included the random animal genetic effect and fixed effects of the contemporary groups (herd-year-month of test-day), age of cow (linear and quadratic effects), and days in milk (linear effect). The residual covariance matrix was assumed to have full rank. Moreover, 2 random regression models were applied. Variance components were estimated by restricted maximum likelihood method. The heritability estimates ranged from 0.11 to 0.24 . The genetic correlation estimates between TD obtained with the PC2 model were higher than those obtained with the MV model, especially on adjacent test-days at the end of lactation close to unity. The results indicate that for the data considered in this study, only 2 principal components are required to summarize the bulk of genetic variation among the 10 traits.

Key words: factor analytic model, multivariate analysis, reduced rank model

\section{Short Communication}

The test-day model (TDM) has been suggested as the method of choice for the analysis of repeated measurements of milk yield traits (Pander et al., 1992; Schaeffer and Jamrozik, 1996). The use of daily production with the test-day model has several advantages over the traditional procedures of evaluating lactation

Received April 28, 2011.

Accepted December 19, 2011.

${ }^{1}$ Corresponding author: annaizabb@hotmail.com records, such as the ability to account for environmental effects on each test-day and to model individual lactation curves (Schaeffer et al., 2000). Moreover, it has the advantage of allowing the use of all available information for genetic evaluation of dairy cattle (Jensen, 2001). Nonetheless, TDM may face some computational burden in analyzing large data sets, as it requires estimation of many more parameters compared with the standard 305-d lactation model. Different statistical models have been proposed to model longitudinal data. In the repeatability model, the records are considered as repeated expressions of the same trait and the genetic correlations between the test-day are assumed to be close to unity, which may not be true. Test-day records can also be analyzed by a full multivariate model in which each test-day milk yield within lactation is considered as a distinct trait, assuming an unstructured covariance matrix between records. The most widely used model is the random regression model, which models the change in covariance with DIM. In general, high-degree polynomials are necessary to model the covariance structure, and problems in adjusting the beginning and end of the lactation curve are common (El Faro et al., 2008; Bohmanova et al., 2009; Bignardi et al., 2009a).

Today, principal components and factor analyses provide powerful means to model multivariate covariance matrices, reducing the number of parameters to be estimated. In addition, they can substantially reduce computational requirements of mixed model analyses compared with standard multivariate models (Meyer, 2009).

Meyer and Kirkpatrick (2005) stated that principal component analyses present advantages compared with a traditional multivariate approach. First, standard methods require estimation of a large number of parameters; with $q$ traits, the number of parameters is $q(q+1) / 2$, whereas estimating the first $m$ principal components reduces the number of parameters to $m(2 q$ $-m+1) / 2$. Second, sampling variances tend to in- 
crease with the number of parameters estimated. If the principal components omitted have eigenvalues close to zero, biases in estimates due to this omission are negligible and can be outweighed by reduced sampling variances, yielding smaller mean square errors than full rank analyses. Third, the principal component analyses reduce computational requirements when comparable to an $m$-variate, full-rank analysis. This may facilitate analyses of larger data sets. Factor analysis is related to principal component analysis but not identical. A major result of a factor analysis is the splitting of the total variance of each variable into its common and unique components, which are also called the communality and the uniqueness of a variable, respectively. The unique variance consists of specific variance and random error that usually cannot be separated. The derived common factors are the largest, statistically independent (uncorrelated or orthogonal) patterns of relationships among the variables. The factors are defined by loadings that measure which variables are involved in what factor and to what degree (Enevoldsen et al., 1996).

The purpose of this work was to estimate genetic parameters for test-day milk yield using different models in first-lactation Holsteins. Principal components and factor analytic models were applied and results were compared with those obtained by a classical multivariate and a random regression model.

A total of 46,089 individual monthly test-day (TD) milk yields (10 test-days), from 7,331 complete first lactations of Holstein cattle were analyzed. The data were obtained from the Herd Analyses and Milk Quality Program carried out by Clínica do Leite (Milk Quality Laboratory of Luiz de Queiroz Agriculture School, ESALQ-USP). The cows were descendants of 492 sires, and were distributed over 93 herds located in the southeastern region of Brazil, with age at first calving varying from 18 to $48 \mathrm{mo}$. Test-day records were between d 5 and 305 of lactation, recorded from 1995 to 2003. The contemporary groups were defined as herd-yeartest-day, with the restriction that each group contained at least 4 animals. The relationship matrix had a total of 17.688 animals. The structure of the data set after editing is summarized in Table 1.

A standard multivariate analysis (MV), random regression model (RRM), reduced rank analyses fitting the first 2, 3, and 4 genetic principal components (PC), and analyses that fitted a factor analytic structure (FAS) considering 2, 3, and 4 factors were applied. The models included the random animal genetic effect and fixed effects of contemporary groups, age of cow (linear and quadratic effects), and DIM (linear effect). The residual covariance matrix was assumed to have full rank.
The matrix representation of the multivariate model is

$$
\mathbf{y}=\mathrm{X} \boldsymbol{\beta}+\mathbf{Z u}+\mathbf{e}
$$

with $\mathbf{y}$ the vector of observations for 10 traits (testdays); $\boldsymbol{\beta}, \mathbf{u}$, and $\mathbf{e}$ vectors of fixed effects, random effects, and residuals, respectively; and $\mathbf{X}$ and $\mathbf{Z}$ the design matrices pertaining to $\boldsymbol{\beta}$ and $\mathbf{u}$. Assume $\mathbf{u}$ represents additive genetic effects only for $\mathrm{N}$ individuals, with covariance matrix $\operatorname{Var}(\mathrm{u})=\Sigma \otimes \mathbf{A}$, and $\mathbf{A}$ is the numerator relationship matrix and $\operatorname{Var}(\mathrm{e})=\mathrm{R}$. The corresponding mixed model equations for a standard MV analysis are

$$
\left(\begin{array}{cc}
X^{\prime} R^{-1} X & X^{\prime} R^{-1} Z \\
Z^{\prime} R^{-1} X & Z^{\prime} R^{-1} Z+\sum^{-1} \otimes A^{-1}
\end{array}\right)\left(\begin{array}{l}
\hat{\beta} \\
\hat{u}
\end{array}\right)=\left(\begin{array}{c}
X^{\prime} R^{-1} y \\
Z^{\prime} R^{-1} y
\end{array}\right) \cdot[2]
$$

Reparameterizing equation [1]:

$$
\begin{gathered}
\mathbf{y}=\mathrm{X} \boldsymbol{\beta}+\mathbf{Z}^{*} \mathbf{u}^{*}+\mathbf{e}, \\
\text { with } \mathbf{Z}^{*}=\mathbf{Z}(\mathbf{E} \otimes \mathbf{I}), \mathbf{u}^{*}=\left(\mathbf{E}^{\prime} \otimes \mathbf{I}\right), \\
\operatorname{Var}\left(\mathbf{u}^{*}\right)=(\mathbf{I} \otimes \mathbf{A}),
\end{gathered}
$$

yields an equivalent model, which fits genetic values for the principal components (PC). $\Sigma=\mathbf{E} \boldsymbol{\Lambda} \mathbf{E}^{\prime}$ denotes the eigenvalue decomposition of the matrix of genetic covariances, with $\boldsymbol{\Lambda}$ the diagonal matrix of eigenvalues, and $\lambda_{i}$ and $\mathbf{E}$ the corresponding matrix of eigenvectors, and $e_{i}$ is $\mathbf{E E}^{\prime}=\mathbf{I}$, where $\mathbf{I}$ is the identity matrix. Assume that $\lambda_{i}$ and $e_{i}$ are ordered in descending order of magnitude of $\lambda_{i}$. To consider only the leading $m$ genetic $\mathrm{PC}$, replace $\mathbf{E}$ with $\mathbf{E}_{\mathrm{m}}$, the $10 \times m$ matrix comprising the first $m$ columns of $\mathbf{E}, e_{1}, \ldots, e_{m}$. This gives $\mathbf{Z}^{*}$ with number of columns proportional to $m$ rather than 10 . The number of equations in equation [3] is reduced correspondingly (replacing $\boldsymbol{\Lambda}$ by its submatrix $\boldsymbol{\Lambda}_{m}$, consisting of the first $m$ rows and columns), and $\mathbf{u}^{*}$ contains $m$ elements for each individual (Meyer and Kirkpatrick, 2005). The mixed model equations are

Table 1. Summary of data structure

\begin{tabular}{lc}
\hline Information & Statistics \\
\hline Records (no.) & 46,089 \\
Animals with records (no.) & 7,331 \\
Sires (no.) & 492 \\
Dams (no.) & 6,035 \\
Overall mean milk yield (kg) & 26.63 \\
SD of milk yield (kg) & 7.50 \\
\hline
\end{tabular}


$\left(\begin{array}{cc}X^{\prime} R^{-1} X & X^{\prime} R^{-1} Z^{*} \\ \left(Z^{*}\right)^{\prime} R^{-1} X & \left(Z^{*}\right)^{\prime} R^{-1} Z^{*}+\Lambda^{-1} \otimes A^{-1}\end{array}\right)\left(\begin{array}{c}\hat{\beta} \\ \hat{u}^{*}\end{array}\right)=\left(\begin{array}{c}X^{\prime} R^{-1} y \\ \left(Z^{*}\right)^{\prime} R^{-1} y\end{array}\right)$.

In a factor analytic model (FA), genetic effects are separated into $m$ common $(c)$ and $q$ specific $(s)$ factors. Covariance matrix can be written $\Sigma=\boldsymbol{\Gamma} \boldsymbol{\Gamma}^{\prime}+\boldsymbol{\Psi}$, with the number of columns of $\boldsymbol{\Gamma}$ equal to the number of factors considered and $\boldsymbol{\Psi}$ a diagonal matrix. The matrix representation of the model is

$$
\mathbf{y}=\mathbf{X b}+\mathbf{Z}^{+} \mathbf{c}+\mathbf{Z}^{\mp} \mathbf{s}+\mathbf{e}
$$

with $c$ and $s$ representing the common and specific factors, respectively. Assuming $\mathrm{Z}^{+}=\mathrm{Z}(\Gamma \otimes \mathrm{I}), \mathrm{u}^{+}=(\Gamma \otimes$ I) $c+s, c=\left(I_{N} \otimes \Gamma^{\prime} \Sigma^{-1}\right) u, s=\left(I_{N} \otimes \Psi^{\prime} \Sigma^{-1}\right) u, \operatorname{Var}(c)=$ I, and $\operatorname{Var}(s)=\Psi \otimes \mathrm{I}$ (Thompson et al., 2003; Meyer, 2009). The corresponding mixed model equations are

$\left(\begin{array}{ccc}X^{\prime} R^{-1} X & X^{\prime} R^{-1} Z^{+} & X^{\prime} R^{-1} Z^{\mp} \\ Z^{+}+R^{-1} X & Z^{+} \cdot R^{-1} Z^{+}+I_{M} \otimes A^{-1} & Z^{+} \cdot R^{-1} Z^{\mp} \\ Z^{\mp} \cdot R^{-1} X & Z^{\mp} \cdot R^{-1} Z^{+} & Z^{\mp} \cdot R^{-1} Z^{\mp}+\psi^{-1} \otimes A^{-1}\end{array}\right)$ $\left(\begin{array}{c}\hat{\beta} \\ \hat{c} \\ \hat{s}\end{array}\right)=\left(\begin{array}{c}X^{\prime} R^{-1} y \\ Z^{+} \cdot R^{-1} y \\ Z^{\mp} \cdot R^{-1} Y\end{array}\right)$.

Two RRM, as described by Bignardi et al. (2009a), were applied. First, sixth- and seventh-order Legendre polynomials were assumed for direct additive genetic and permanent environmental effects, respectively, modeling the residual variances structure with 6 classes (6.7.het6). Second, the same model, imposing rank restrictions (principal components analysis) on estimated

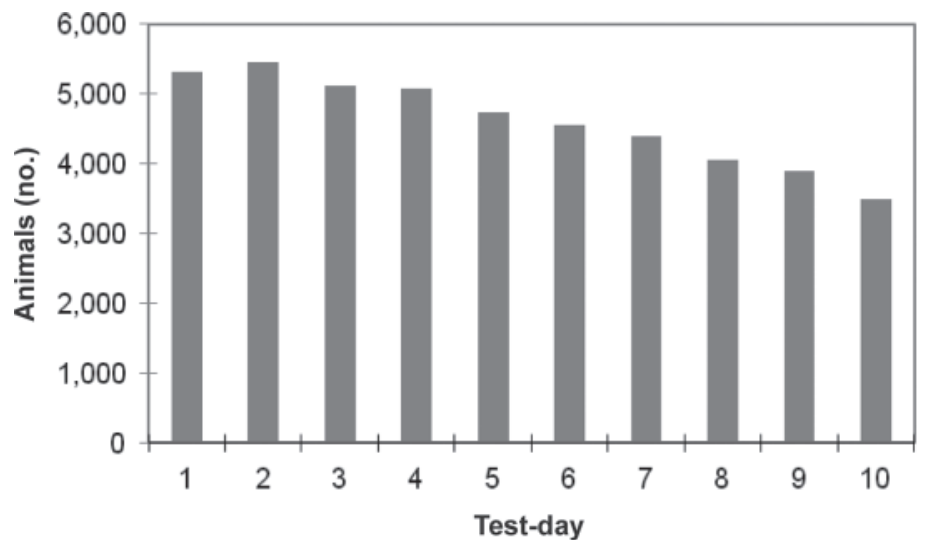

Figure 1. Number of animals (left) and milk yield (right). covariance functions, was also applied (6.7.r.het6). For RRM, fixed effects were contemporary groups and age of cow (linear and quadratic covariables). Mean trends were modeled by a cubic regression on Legendre orthogonal polynomials of DIM.

Variance components were estimated by REML, using the Wombat package (Meyer, 2007a). Models were compared by Akaike's information criterion (AIC) and Schwarz's Bayesian information criterion (BIC), as reported by Wolfinger (1993). The AIC and BIC allow the comparison between non-hierarchical models and penalize those with a larger number of parameters, with the BIC attributing a more rigorous penalty. Spearman correlation coefficients between predicted breeding values obtained in different analyses were calculated.

\section{RESULTS AND DISCUSSION}

The number of records at each test-day and the corresponding means of milk yield are shown in Figure 1. The peak period of milk yield was observed from TD3 to TD5. After this period, milk yield decreased with increasing DIM as well as the number of records. The standard deviation of milk yield ranged from $7.0 \mathrm{~kg}$ (TD3) to $8.3 \mathrm{~kg}$ (TD10).

A summary of the results in terms of alternative model comparison criteria is shown in Table 2. According to all information criteria results, both RRM, with or without the reduced rank analysis, provided the worst fit, followed by the MV. The BIC values for $\mathrm{PC}$ and FAS models improved with declining number of parameters and were lower for PC models, probably due to the reduced number of parameters. Based on AIC, the best model was PC3. However, the BIC criterion, which penalizes more rigorously models with a larger number of parameters, indicated PC2 as the best. Meyer (2007b), using a multivariate model, factor

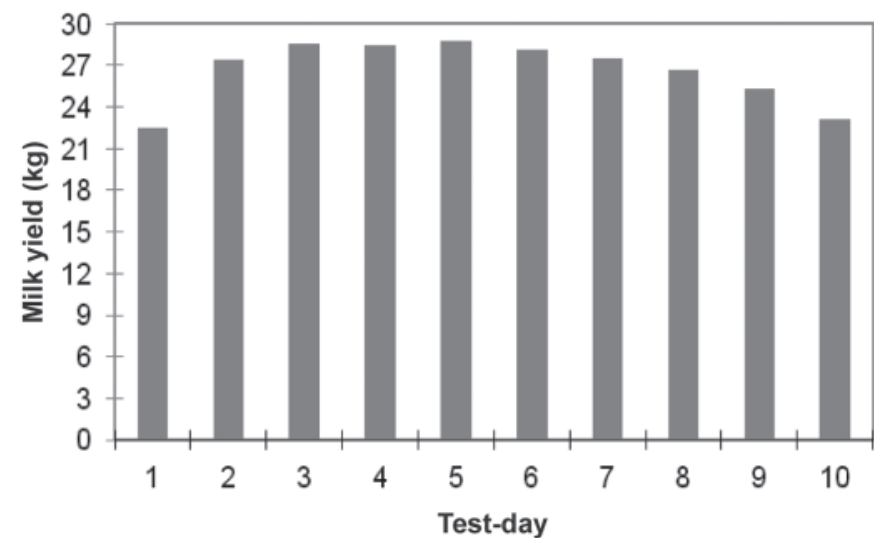


Table 2. Models, number of parameters (p) for different analyses together with the maximum log-likelihood ( $\log$ L), Akaike's information criterion (AIC) and Schwarz's Bayesian information criterion (BIC), all scaled as deviations from the multivariate model

\begin{tabular}{lrccc}
\hline Model $^{1}$ & $\mathrm{p}$ & $\log \mathrm{L}$ & $\mathrm{AIC}$ & $\mathrm{BIC}$ \\
\hline PC2 & 74 & $-59,284.19$ & $118,716.38$ & $119,324.83$ \\
PC3 & 82 & $-59,276.23$ & $118,716.46$ & $119,390.69$ \\
PC4 & 89 & $-59,271.84$ & $118,721.68$ & $119,453.46$ \\
MV & 110 & $-59,269.59$ & $118,759.18$ & $119,663.64$ \\
FAS2 & 84 & $-59,280.77$ & $118,729.54$ & $119,420.22$ \\
FAS3 & 92 & $-59,275.14$ & $118,734.28$ & $119,490.74$ \\
FAS4 & 99 & $-59,271.57$ & $118,741.14$ & $119,555.15$ \\
6.7.het6 & 55 & $-90,218.12$ & $180,546.25$ & $181,021.60$ \\
6.7.r.het6 & 39 & $-90,325.25$ & $180,637.75$ & $181,017.30$ \\
\hline
\end{tabular}

${ }^{1} \mathrm{PCn}=$ analysis fitting the leading $\mathrm{n}$ principal components; $\mathrm{MV}=$ multivariate model; FASn = analysis fitting a factor analytic model with $\mathrm{n}$ factors; 6.7 .het $6=$ sixth- and seventh-order Legendre polynomials were assumed for direct additive genetic and permanent environmental effects, respectively, modeling the residual variances structure with 6 classes; and 6.7.r.het6: sixth- and seventh-order Legendre polynomials were assumed for direct additive genetic and permanent environmental effects, respectively, imposing rank restrictions, modeling the residual variances structure with 6 classes.

analysis, and principal component analysis to study 14 carcass traits of Angus cattle, observed that principal components provided the best fit for the genetic covariance structure compared with the other approaches, in agreement with this study. Compared with MV, the PC2 model showed an expressive reduction in the number of parameters $(\mathrm{n}=36)$. Moreover, the $\mathrm{PC} 2$ model converged much faster and reduced the number of equations in the mixed model by $88 \%$ compared with MV. According to Meyer and Kirkpatrick (2005), computational requirements in mixed model analyses generally increase dramatically with the number of equations, and even a small reduction in the number of $\mathrm{PC}$ fitted can have a dramatic effect on the efficiency of analyses. Meyer (2009) also reported that PC models converged faster than MV and FAS models.

Only results for the 2 best models were compared with those from the MV model. In general, eigenvalues (Figure 2) from the 3 models (MV, PC2, and FAS2) were similar. The genetic eigenvalues tended to be slightly larger when fitting a smaller number of principal components or factors.

The estimates of phenotypic variances were similar for all models and were higher at the end of lactation (Figure 3). Phenotypic variances ranged from 28.09 to $36.90 \mathrm{~kg}^{2}$ and were similar to those reported by Bignardi et al. (2008) for Holstein cattle using standard bi-trait analyses of 10 test-day milk yield records. The highest genetic variances estimated with the 3 models were observed at the end of lactation. In general, at this stage, a smaller number of records is available, which may cause difficulty in model fitting. The genetic variances obtained from the 3 models were similar (Figure $3)$. Additive genetic variances were always lower than residual variances and were constant throughout the lactation period, similar to those reported by Jamrozik and Schaeffer (1997) and Olori et al. (1999) for Holstein cattle using RRM. In FAS models, genetic variances comprise common and specific factors genetic separately. In all FAS models, the specific variances obtained were responsible for only a small portion of

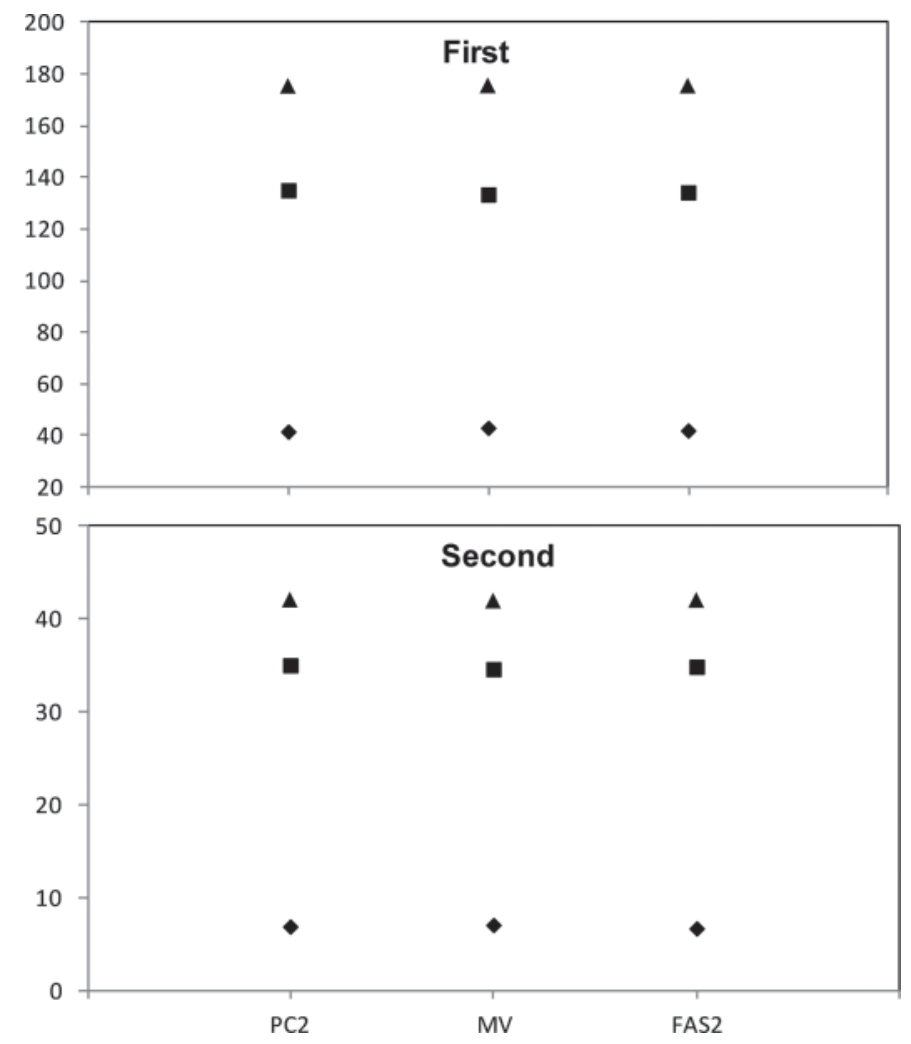

Figure 2. Estimates of the first 2 eigenvalues of genetic ( ), residual $(\mathbf{\square})$, and phenotypic $(\boldsymbol{\Delta})$ covariance matrices from a full rank multivariate analysis (MV), model fitting the leading 2 principal components (PC2), and model fitting a factor analytic model with 2 factors (FAS2). 
MULTIVARIATE ANALYSES OF MILK YIELD
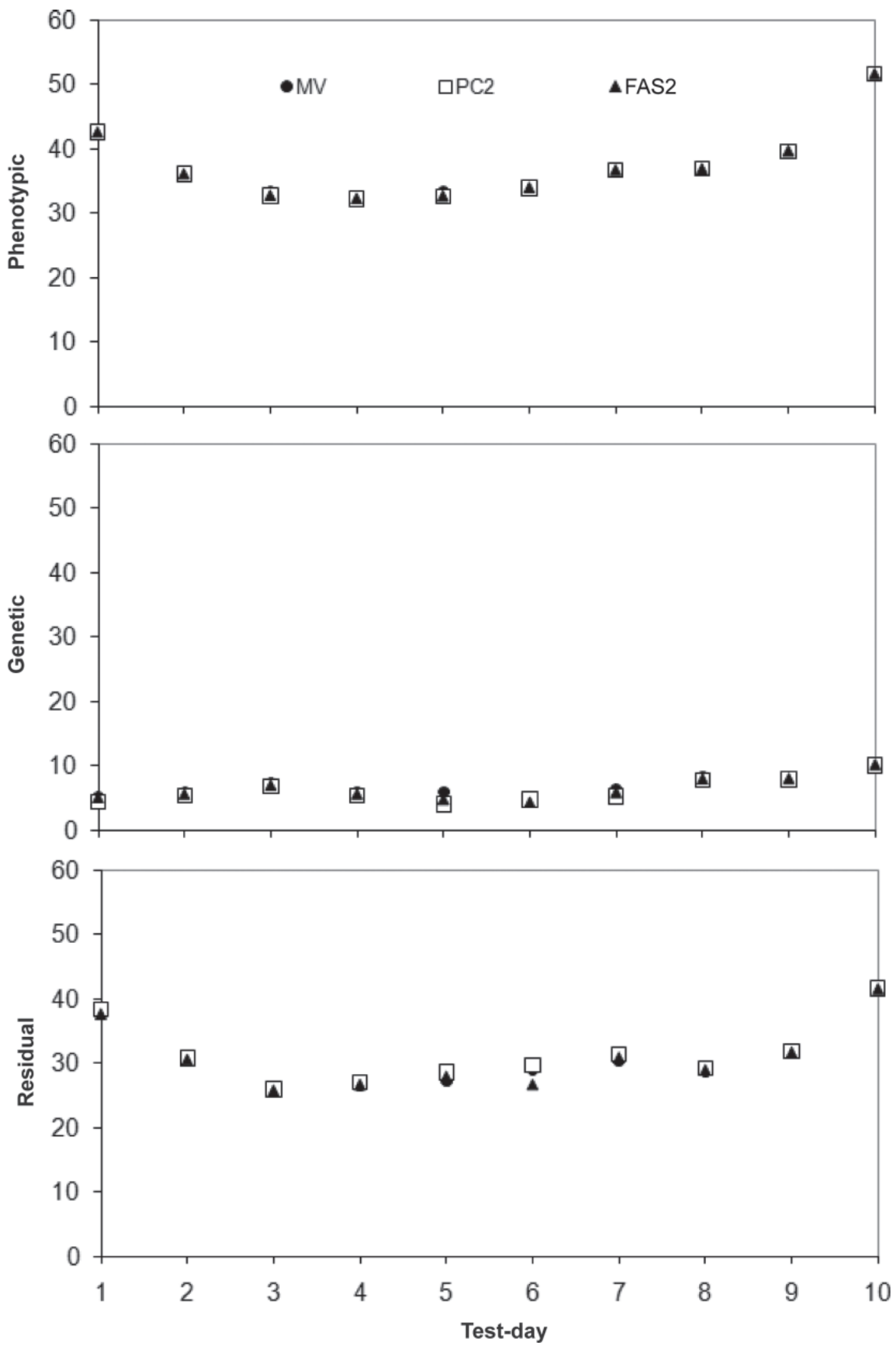

Figure 3. Phenotypic, genetic, and residual variances estimated by the multivariate model (MV), model fitting the leading 2 principal components (PC2), and model fitting a factor analytic model with 2 factors (FAS2). 
Table 3. Heritability estimates ( \pm SE) obtained by multivariate model $(\mathrm{MV})$, analysis fitting the leading 2 principal components (PC2), and model fitting a factor analytic model with 2 factors (FAS2)

\begin{tabular}{lccc}
\hline Test-day & MV & PC2 & FAS2 \\
\hline 1 & $0.14( \pm 0.03)$ & $0.11( \pm 0.03)$ & $0.11( \pm 0.03)$ \\
2 & $0.16( \pm 0.04)$ & $0.14( \pm 0.03)$ & $0.14( \pm 0.04)$ \\
3 & $0.21( \pm 0.04)$ & $0.19( \pm 0.04)$ & $0.20( \pm 0.04)$ \\
4 & $0.17( \pm 0.04)$ & $0.16( \pm 0.04)$ & $0.15( \pm 0.04)$ \\
5 & $0.14( \pm 0.04)$ & $0.11( \pm 0.03)$ & $0.12( \pm 0.04)$ \\
6 & $0.15( \pm 0.04)$ & $0.11( \pm 0.03)$ & $0.12( \pm 0.04)$ \\
7 & $0.16( \pm 0.04)$ & $0.14( \pm 0.04)$ & $0.14( \pm 0.04)$ \\
8 & $0.21( \pm 0.05)$ & $0.20( \pm 0.05)$ & $0.20( \pm 0.05)$ \\
9 & $0.19( \pm 0.05)$ & $0.18( \pm 0.05)$ & $0.19( \pm 0.05)$ \\
10 & $0.24( \pm 0.05)$ & $0.23( \pm 0.05)$ & $0.20( \pm 0.06)$ \\
\hline
\end{tabular}

the genetic variation. For model FA2, the specific variances accounted for only $0.03 \%$ of the genetic variation observed on TD1, and these values were practically zero on the other test-days. According to Meyer (2009), if specific factors are assumed absent, the FAS model will be reduced to a PC model. Because, in the present study, the specific factors of the FAS models were practically zero, the use of these models would not be advantageous. The residual variance estimates presented a trend similar to that of phenotypic variance, with small oscillations being observed between estimates obtained in the middle of lactation by the 3 models.

The heritability estimates (Table 3) obtained with the MV model were higher than those with $\mathrm{PC} 2$ and FAS2 models, as expected, because the genetic variances estimated with the MV model were higher than those obtained with both reduced rank models. Estimates of heritability increased until TD3, remained constant and then increased from TD8 through the end of lactation. These results do not agree with those from several studies (Druet et al., 2003; Gengler et al., 2005; Shadparvar and Yazdanshenas, 2005; Silvestre et al., 2006; Bilal et al., 2008) in which test-day milk yield heritability estimates were low during early and late lactation, but were similar to those reported by El Faro and Albuquerque (2003) and Bignardi et al. (2008) in Brazil. Heritability estimates did not differ between PC2 and FAS2 and were similar to those reported for
Holstein cattle in Brazil using RRM (Cobuci et al., 2005; Araújo et al., 2006; Melo et al., 2007; Bignardi et al., 2009a,b) and standard TDM (Melo et al., 2005; Bignardi et al., 2008).

The genetic effects in mid lactation were less correlated than those at the end of lactation (Table 4). The genetic correlation estimates between TD obtained with the PC2 model were higher than those obtained with the MV model, especially on adjacent test-days at the end of lactation close to unity. Except for the estimated correlations between TD1 and at the end of lactation (TD8, TD9, and TD10), all values were above 0.50 , indicating that selection for increased milk yield in a given period of lactation may have a positive influence on the remaining period of lactation. These results agree with those reported by Rekaya et al. (1999) and Olori et al. (1999) and in Brazil by Cobuci et al. (2005) for Holstein cattle using RRM. The phenotypic correlations obtained with the MV and $\mathrm{PC} 2$ models (not shown) showed the same trend as the genetic correlations, with higher correlations being observed between adjacent test-days. The phenotypic and residual correlations estimates were lower than the genetic correlations. The correlation estimates obtained by FAS2 (not shown) are very similar to those of the PC2 model.

Table 5 shows the Spearman correlation coefficients between predicted breeding values for test-day milk yields estimated with the MV and PC2 models for

Table 4. Estimates of genetic correlations for test-day milk yield (TD1 to TD10) obtained by multivariate model (above the diagonal) and the differences between the model fitting the leading 2 principal components and the multivariate model correlations (PC2-MV) (below the diagonal)

\begin{tabular}{|c|c|c|c|c|c|c|c|c|c|c|}
\hline & TD1 & TD2 & TD3 & TD4 & TD5 & TD6 & TD7 & TD8 & TD9 & TD10 \\
\hline TD2 & 0.16 & - & 0.95 & 0.87 & 0.82 & 0.63 & 0.63 & 0.67 & 0.57 & 0.51 \\
\hline TD3 & 0.16 & 0.04 & - & 0.96 & 0.81 & 0.71 & 0.76 & 0.78 & 0.71 & 0.65 \\
\hline TD5 & 0.06 & -0.01 & 0.09 & 0.14 & - & 0.86 & 0.82 & 0.89 & 0.83 & 0.82 \\
\hline TD6 & 0.12 & -0.06 & -0.01 & 0.04 & 0.08 & - & 0.87 & 0.82 & 0.91 & 0.78 \\
\hline TD7 & -0.01 & 0.06 & 0.04 & 0.15 & 0.16 & 0.12 & - & 0.94 & 0.96 & 0.88 \\
\hline TD10 & -0.01 & 0.10 & 0.08 & 0.09 & 0.13 & 0.22 & 0.12 & 0.05 & 0.09 & - \\
\hline
\end{tabular}


Table 5. Spearman correlation coefficients between breeding values for test-day milk yields (TD1 to TD10) obtained by multivariate model (MV) and analysis fitting the leading 2 principal components (PC2)

\begin{tabular}{lc}
\hline $\begin{array}{l}\text { Test-day } \\
\text { milk yield }\end{array}$ & MV-PC2 \\
\hline TD1 & 0.87 \\
TD2 & 0.92 \\
TD3 & 0.96 \\
TD4 & 0.95 \\
TD5 & 0.93 \\
TD6 & 0.93 \\
TD7 & 0.95 \\
TD8 & 0.97 \\
TD9 10 & 0.97 \\
\hline
\end{tabular}

all sires in the data set $(n=492)$. Most of the rank correlations between breeding values for test-day milk yields obtained with MV and PC were high, above 0.87 , indicating that the same sires would be selected independent of the model.

The results indicate that for the data set considered in this study, both principal components and factor analytic approaches can provide more parsimonious models compared with an MV. Furthermore, these models allow a reduction in the number of parameters to be estimated without causing any significant reduction of the goodness of fit. On the other hand, because the specific factors of the FAS models were practically zero in the present study, the use of these models would not be advantageous over the PC. Only 2 principal components are required to summarize the bulk of genetic variation among the 10 traits, resulting in a reduction in dimension of the mixed model analyses and a decrease in computational requirements.

\section{ACKNOWLEDGMENTS}

This work was funded by Fundação de Amparo à Pesquisa do Estado de São Paulo (FAPESP) and Coordenação de Aperfeiçoamento de Pessoal de Nível Superior (Capes), Brazil.

\section{REFERENCES}

Araújo, C. V., R. A. Torres, C. N. Costa, R. A. Torres Filho, S. I. Araújo, P. S. Lopes, A. J. Regazzi, C. S. Pereira, and J. L. R. Sarmento. 2006. Random regressions models to describe the genetic variation of milk yield in Holstein breed. R. Bras. Zootec. 35:975-981.

Bignardi, A. B., L. El Faro, L. G. Albuquerque, V. L. Cardoso, and P. F. Machado. 2008. Finite dimension models to estimate genetic parameters for first-lactation milk yields of Holstein cows. Ciencia Rural 38:1705-1710.

Bignardi, A. B., L. El Faro, L. G. Albuquerque, V. L. Cardoso, and P. F. Machado. 2009a. Random regression models to estimate testday milk yield genetic parameters Holstein cows in southeastern Brazil. Livest. Sci. 123:1-7.
Bignardi, A. B., L. El Faro, L. G. Albuquerque, V. L. Cardoso, and P. F. Machado. 2009b. Parametric correlation functions to model the structure of permanent environmental (co)variances in milk yield random regression models. J. Dairy Sci. 92:4634-4640.

Bilal, G., M. S. Khan, I. R. Bajwa, and M. Shafiq. 2008. Genetic control of test-day milk yield in Sahiwal cattle. Pakistan Vet. J. 28:21-24.

Bohmanova, J., F. Miglior, and J. Jamrozik. 2009. Use of test-day records beyond three hundred five days for estimation of three hundred five-day breeding values for production traits and somatic cell score of Canadian Holsteins. J. Dairy Sci. 92:5314-5325.

Cobuci, J. A., R. F. Euclydes, P. S. Lopes, C. N. Costa, R. A. Torres, and C. S. Pereira. 2005. Estimation of genetic parameters for test-day milk in Holstein cows using a random regression model. Genet. Mol. Biol. 28:75-83.

Druet, T., F. Jaffrezik, D. Boichard, and V. Ducrocq. 2003. Modeling lactation curves and estimation of genetic parameters for first lactation test-day records of French Holstein cows. J. Dairy Sci. 86:2480-2490.

El Faro, L., and L. G. Albuquerque. 2003. Estimation of genetic parameters for first lactation test-day records and total milk yield for Caracu cows. Rev. Bras. Zootecn. 32:284-294.

El Faro, L., L. G. Albuquerque, and V. L. Cardoso. 2008. Variance component estimates for test-day milk yield applying random regression models. Genet. Mol. Biol. 33:665-673.

Gengler, N., G. R. Wiggans, and A. Gillon. 2005. Adjustment for heterogeneous covariance due to herd milk yield by transformation of test-day random regressions. J. Dairy Sci. 88:2981-2990.

Enevoldsen,, C., J. Hindhede, and T. Kristensen. 1996. Dairy herd management types assessed from indicators of health, reproduction, replacement, and milk production. J. Dairy Sci. 79:12211236.

Jamrozik, J., and L. R. Schaeffer. 1997. Genetic evaluation of dairy cattle using test-day yields and random regression model. J. Dairy Sci. 80:1217-1226.

Jensen, J. 2001. Genetic evaluation of dairy cattle using test-day models. J. Dairy Sci. 84:2803-2812.

Melo, C. M. R., C. N. Costa, U. I. Packer, and P. F. Machado. 2005. Genetic parameters for test day and lactation milk yields of first lactation Holstein cows. R. Bras. Zootec. 34:796-806.

Melo, C. M. R., I. U. Packer, C. N. Costa, and P. F. Machado. 2007. Genetic parameters for test day milk yields of first lactation Holstein cows by random regression models. Animal 1:325-334.

Meyer, K. 2007a. WOMBAT: A tool for mixed model analyses in quantitative genetics by restricted maximum likelihood (REML). J. Zhejiang Univ. Sci. B 8:815-821.

Meyer, K. 2007b. Multivariate analyses of carcass traits for Angus cattle fitting reduced rank and factor analytic models. J. Anim. Breed. Genet. 124:50-64.

Meyer, K. 2009. Factor-analytic models for genotype $\times$ environment type problems and structured covariance matrices. Genet. Sel. Evol. 41:21-32

Meyer, K., and M. Kirkpatrick. 2005. Restricted maximum likelihood estimation of genetic principal components and smoothed covariance matrices. Genet. Sel. Evol. 37:1-30.

Olori, V. E., W. G. Hill, B. J. Mcguirk, and S. Brotherstone. 1999. Estimating variance components for test day milk records by restricted maximum likelihood with a random regression animal model. Livest. Prod. Sci. 61:53-63.

Pander, B. L., W. G. Hill, and R. Thompson. 1992. Genetic parameters of day records of British Holstein-Friesian heifers. Anim. Prod. 55:11-21.

Rekaya, R., M. J. Carabano, and M. A. Toro. 1999. Random regression in animal models for test-day production in dairy cattle. Proc. 5th World Congr. Genet. Appl. Livest. Prod. 18:443-446.

Schaeffer, L. R., and J. Jamrozik. 1996. Multiple-trait prediction of lactation yields for dairy cows. J. Dairy Sci. 79:2044-2055.

Schaeffer, L. R., J. Jamrozik, R. Van Dorp, D. F. Keltonb, and D. W. Lazenbyc. 2000. Estimating daily yields of cows from different milking schemes. Livest. Prod. Sci. 65:219-227. 
Shadparvar, A. A., and M. S. Yazdanshenas. 2005. Genetic parameters of milk yield and milk fat percentage test-day records of Iranian Holstein cows. Asian-australas. J. Anim. Sci. 18:1231-1236.

Silvestre, A. M., F. Petim-Batista, and J. Colaço. 2006. The accuracy of seven mathematical functions in modeling dairy cattle lactation curves based on test-day records from varying sample schemes. J. Dairy Sci. 89:1813-1821.
Thompson, R., B. R. Cullis, A. B. Smith, and A. R. Gilmour. 2003. A sparse implementation of the Average Information algorithm for factor analytic and reduced rank variance models. Aust. N. Z. J. Stat. 45:445-459.

Wolfinger, R. D. 1993. Covariance structure selection in general mixed models. Commun. Stat. Simul. C 22:1079-1106. 